Original Article

\title{
Role of focused Ultrasound in the diagnosis of acute appendicitis in Pediatric population. Selection of a standard diagnostic criterion Sayani $R^{l}$, Mustahsan SM ${ }^{2}$, Ali AAM ${ }^{2}$, Anwar M ${ }^{3}$
}

Interventional Radiologist, Dept of Radiology, Aga Khan University Hospital

${ }^{2}$ Final year medical students, Sindh Medical College, Dow University of Health Sciences

${ }^{3}$ Pediatric Radiologist, Dept of Radiology, Aga Khan University Hospital

Objective To evaluate the diagnostic role of focused ultrasound of right iliac fossa in clinically suspected cases of acute appendicitis in pediatric population and its value as a standard diagnostic tool in that age group Material and Method A retrospective, cohort study was conducted at radiology department of Agha Khan University involving 239 children less than 16 years with clinically suspected appendicitis referred to radiology department from January 2009 to October 2011 were included. Medical records were reviewed for patient demographics, presenting signs and symptoms, sonographic, surgical findings and histopathological results. Results Two hundred and thirty nine patients with clinical suspicion of appendicitis were referred for focused ultrasound for appendix. Ultrasound examination showed 59 cases $(24.6 \%)$ positive for appendicitis and thus they were operated. All of these were positive on histopathology. The remaining 180 cases (75.4\%) that were negative on U/S were managed conservatively. Out of these 180 cases, 13 patients with negative findings on imaging studies were later operated due to clinical decision and/or CT examination. Acute appendicitis was proven in 10 out of the 13 cases. Conclusion Focused ultrasound has high sensitivity in acute appendicitis for the assessment of clinically equivocal cases of pediatrics population. It can be used as an initial screening modality especially in pediatric population, thus reducing additional cost and radiation risk associated with CT scans and also reducing rate of negative surgery.

\section{KEYWORDS}

Focused ultrasound, acute appendicitis, pediatric population, CT Scan

\section{INTRODUCTION}

Acute appendicitis is one of the most common causes of acute abdominal pain in all age groups and is considered to be the most frequent condition leading to emergent abdominal surgery in children due to risk of rupture. An early diagnosis of the condition followed by appendectomy, before complications like gangrene or perforation develops, is the main factor defining the success rate. Although appendicitis occurs rarely in young children, this group can be particularly challenging to diagnose due to atypical clinical presentation, delayed appearance of relevant signs and inability to clearly express their symptoms. In addition, the clinical assessment can be difficult because the child is often troubled and distressed.

Different Imaging modalities like ultrasound (U/S), computed tomography (CT) and magnetic resonance imaging (MRI) are useful in assisting the diagnosis of appendicitis especially in clinically suspicious cases. The conclusions drawn from different past comparative studies show standardized CT scan to be the modality of choice for diagnosis of appendicitis in general population due to its higher accuracy than U/S. However the use of ionizing radiation and intravenous or gastrointestinal contrast material make it a relatively more invasive test which negates its use as an ideal choice in pediatric population. U/S on the other hand is simple, easily available, noninvasive, convenient and cost effective. Moreover U/S in pediatric population cannot only identify inflamed appendices but also gauge the severity of disease. (Kaneko, K., 2004)

The aim of this study was to determine the role of focused ultrasound in children presenting with clinically suspected acute appendicitis. We have selected the Ultrasound as the diagnostic modality of choice because of its wide availability, simplicity, low cost, and noninvasiveness and its value as a standard choice for diagnosis of acute appendicitis in suspected pediatric cases.

\section{METHODOLOGY}

A retrospective cohort study was conducted at Radiology Department of Aga Khan University Hospital, Karachi on patients presenting with the clinical signs and symptoms suggestive of acute appendicitis. The data was obtained from medical records of 239 children (age less than 16 years) from
January 2009 to October 2011. The clinically suspected patients for appendicitis underwent ultrasound. Based on clinical or ultrasound findings some of these patients also had a focused CT scan preoperatively. The diagnosis was made on the basis of imaging studies which was done by a senior radiology resident and final report was made by consultant radiologist having experience of pediatric-abdominal imaging. The patients that were positive on imaging were operated for appendicitis and histopathological findings were done.

Abdominal Ultrasonography was done by graded compression technique as described by Puylaert. (Karakas, S. P., 2000 \& Akhtar, W., 2011) Criteria for the diagnosis of acute appendicitis. It included identification of a non-compressible, fluid-filled, blind-ended tubularstructure in the right lower quadrant, with sonographic features indicating intestinal origin, and a diameter equal or greater than $6 \mathrm{~mm}$ (Karakas, S. P., 2000 \& Akhtar, W., 2011). CT scan was done by using the multi detector CT scanner (Toshiba aquilion). Scanning was performed from L3 level to pubic symphysis in all the patients. Images were reviewed on reporting console as well as on hard copies (Mardan, M. A.,2007).

Decision for immediate surgery, referral for imaging, observation followed by surgery or imaging, or simply discharge depended on a variety of factors, the foremost being the level of the examiner's experience, and confidence, ,etc., all impossible to standardize in a retrospective study (Brennan, G. D. 2006).

A self-designed Performa was used to collect relevant data of the study subjects from the medical records. The inclusion criteria were the children with clinical signs of acute appendicitis (pain in right iliac fossa, fever, and mass in right iliac fossa), proper vaccination history and without any history of chronic illness where as children with improper health was excluded. SPSS version 20 was used for calculating the results.

The informed verbal consent was taken from the parents and their confidentially were maintained. The study protocol was reviewed by the Ethical Committee Board. 


\section{RESULT}

The mean age of the children who presented with the clinical signs and symptoms of acute appendicitis was $11.24 \pm 3.02$ as shown in table 1 . Total 239 patients were included, $70.7 \%$ were male and $29.3 \%$ were female as shown in table 2 . U/S was done to diagnose or exclude appendicitis in suspected patients. CT scan was also used in $17.2 \%$ cases to help in the diagnosis.

Ultrasound examination showed 59 cases $(24.6 \%)$ positive for appendicitis and thus they were operated. The remaining 180 cases $(75.4 \%)$ that were negative on U/S were managed conservatively. Out of these 180 cases, 13 patients with negative findings on imaging studies were later operated due to clinical decision and/or CT examination. Acute appendicitis was proven in 10 out of the 13 cases. Fifty nine $(81.9 \%)$ out of total 72 patients operated were positive for acute appendicitis on both $\mathrm{U} / \mathrm{S}$ and histopathology as shown by table 3 .

Our study showed $85.5 \%, 100 \%, 100 \%$ and 94.44 sensitivity, specificity, positive and negative predictive values respectively. Also, ultrasonography helped to reduce the rate of negative laparotomies which was $4.1 \%$ in our study which is much lower than the published data.

Table 1: Descriptive Statistics

\begin{tabular}{|l|l|l|l|l|l|}
\hline & $\mathrm{N}$ & Minimum & Maximum & Mean & Std. Deviation \\
\hline Age & 239 & 3 & 16 & 11.24 & 3.025 \\
\hline Valid N (list wise) & 239 & & & & \\
\hline
\end{tabular}

Table 2: Frequency of gender in participants

\begin{tabular}{|l|l|l|l|l|l|}
\hline \multicolumn{2}{|c|}{} & Frequency & Percent & Valid Percent & Cumulative Percent \\
\hline \multirow{3}{*}{ Valid } & F & 70 & 29.3 & 29.3 & 29.3 \\
\cline { 2 - 6 } & M & 169 & 70.7 & 70.7 & 100.0 \\
\cline { 2 - 6 } & Total & 239 & 100.0 & 100.0 & \\
\hline
\end{tabular}

Table 3: Comparative analysis of histopathological and ultrasonographic results in children less than 16 years with clinically suspected appendicitis $(n=72)$

\begin{tabular}{|c|c|c|c|}
\hline \multirow{2}{*}{ Ultrasonography } & \multicolumn{3}{|c|}{ Histopathology } \\
\hline & Negative & Positive & Total \\
\hline Acute appendicitis & 0 & 59 & 59 \\
\hline No Acute appendicitis & 3 & 10 & 13 \\
\hline Total & 3 & 69 & 72 \\
\hline
\end{tabular}

\section{DISCUSSION}

Diagnosis of acute appendicitis is not always straight forward Diagnosis is difficult in pediatric population because $33 \%$ and $50 \%$ will present with atypical presentation so that even the most experienced surgeon may remove a normal appendix or sit on a perforated one resulting in higher rate of negative laparotomies (Brennan, G. D. 2006). Surgery based on clinical decision alone, to avoid the complications of missed or delayed diagnosis in equivocal cases, leads to removal of $15-20 \%$ of normal appendices (Mardan, M. A.,2007). This necessitates the need for a standard diagnostic imaging technique that may facilitate to reduce the rates of negative and unnecessary surgeries. Ultrasound and CT scan are widely used imaging modalities for diagnosing appendicitis, however, their preference over one another as the initial modality of choice remains controversial despite many research studies.

Diagnosing appendicitis by ultrasonography was first described by Puylaert in the 1980s.(Karakas, S. P., 2000 \& Sivit, C. J., 2000) Its usefulness in the diagnosis of acute appendicitis is now established. (Mardan, M. A.,2007) Ultrasonography had generally served as primary imaging modality in children suspected of having appendicitis because it is relatively quick to perform, doesn't involve the use of ionizing radiations and because the abdominal musculature of children in comparison to adults generally has less fat content amplifying its overall accuracy and sensitivity ( Kaiser, S., 2002 \& Sivit, C. J., 2000). However no significant differences were noted in the specificity of ultrasonography between children and adults (Sivit, C. J., 2000).
The 2 largest $(>5,000$ total children $)$ studies found ultrasonography to be $90-92 \%$ sensitive and $97-98 \%$ specific for appendicitis, other studies have noted sensitivities and specificities as low as $80 \%$ and $86 \%$, respectively (Fefferman, N. R.,2001). The sensitivity and specificity of ultrasonography in our research was about $85.5 \%$ and $100 \%$ respectively. These values are similar to higher values of sensitivity and specificity of ultrasonography reported by past studies. Also important finding to note here is that the sensitivity and specificity are of smaller range in pediatric population as compared to adults which is quite assuring for consideration of ultrasonography as the initial imaging modality of choice in diagnosing acute appendicitis in pediatric population. Also ultrasonography helped to reduce the rate of negative laparotomies which was $4.1 \%$ in our study, much lower than the published data (Wagner, P. L.,2008). This also highlights its importance as an efficient tool.

Despite all those benefits associated with ultrasound, many factors have prevented it from becoming standard of care in the diagnostic work-up of appendicitis. These factors include operator dependency, lower accuracy than CT scan, availability, pain, obesity, overlying gas, and perforation (Rothrock, S. G., $2000)$ etc. It could be speculated that the paucity of body fat in younger children may result in increased difficulty in the diagnosis of appendicitis compared with that in adults because of the inability to readily distinguish the appendix from adjacent structures (Sivit, C. J.,2000). Another important factor accounting for the variability in diagnostic accuracy with graded compression sonography is that many sonographic examinations are not performed by radiologists having dedicated expertise with the procedure. At many university hospitals, after-hours 
imaging studies may be obtained and interpreted by residents or fellows. Moreover, many patients with appendicitis are treated at community hospitals and the sonographic examination is performed by a general radiologist. For sonography to be successful on a large scale, it is necessary for it to be performed by radiologists with various degrees of expertise especially in pediatric radiology (Sivit, C. J.,2000)

Recent studies show that CT scan is frequently used in emergency evaluation of pediatric patients with abdominal pain (Fahimi, J., 2012). However it is important to consider the facts here that $\mathrm{CT}$ scan involves exposure to ionizing radiations and use of intravenous or gastrointestinal contrast material make it a relatively more invasive test which is not suitable as initial choice when alternate of ultrasound is available to rule out a significant majority of patients successfully.

Thus it is better to consider less accurate but safer modality of ultrasound as the initial modality of choice for diagnosis of acute appendicitis in children while considering more accurate but risky modality of $\mathrm{CT}$ scan by balancing the future risk of cancer with the use of $\mathrm{CT}$ and the risk of missing positive cases with ultrasonography.

\section{CONCLUSION}

$\mathrm{U} / \mathrm{S}$ is valuable in the diagnosis of appendicitis in children and should remain the method of first choice because it does not involve the use of ionizing radiation. Diagnostic accuracy can be increased significantly by performing $\mathrm{CT}$, which we believe should be added to the imaging protocol for patients who have negative $\mathrm{U} / \mathrm{S}$.

\section{REFERENCES}

1. Kaneko, K., \& Tsuda, M. (2004). Ultrasound-based decision making in the treatment of acute appendicitis in children. Journal of pediatric surgery, 39(9), 1316-1320. Puylaert JBCM (1986) Acute appendicitis: U/S evaluation using graded compression. Radiology 158: $355 \pm 360$

2. Karakas, S. P., Guelfguat, M., Leonidas, J. C., Springer, S., \& Singh, S. P. (2000). Acute appendicitis in children: comparison of clinical diagnosis with ultrasound and CT imaging. Pediatric radiology, 30(2), 94-98

3. Akhtar, W., Ali, S., Arshad, M., Ali, F. N., \& Nadeem, N. (2011). Focused abdominal CT scan for acute appendicitis in children: can it help in need. Journal of the Pakistan Medical Association, 61(5), 474.

4. Brennan, G. D. (2006). Pediatric appendicitis: pathophysiology and appropriate use of diagnostic imaging. CJEM, 8(6), 425

5. Mardan, M. A., Mufti, T. S., Khattak, I. U., Chilkunda, N., Alshayeb, A. A., Mohammad, A. M., \& ur Rehman, Z. (2007). Role of ultrasound in acute appendicitis. J Ayub Med Coll Abbottabad, 19(3), 72-79.

6. Kaiser, S., Frenckner, B., \& Jorulf, H. K. (2002). Suspected Appendicitis in Children: US and CT-A Prospective Randomized Study 1. Radiology, 223(3), 633-638.

7. Sivit, C. J., Dudgeon, D. L., Applegate, K. E., Borisa, V. J., Berlin, S. C., Morrison, S. C., ... \& Grisoni, E. R. (2000). Evaluation of Suspected Appendicitis in Children and Young Adults: Helical CT 1. Radiology, 216(2), 430-433

8. Fefferman, N. R., Roche, K. J., Pinkney, L. P., Ambrosino, M. M., \& Genieser, N. B. (2001). Suspected Appendicitis in Children: Focused CT Technique for Evaluation 1. Radiology, 220(3), 691-695.

9. Wagner, P. L., Eachempati, S. R., Soe, K., Pieracci, F. M., Shou, J., \& Barie, P. S. (2008). Defining the current negative appendectomy rate: for whom is preoperative computed tomography making an impact?. Surgery, 144(2), 276-282.

10. Rothrock, S. G., \& Pagane, J. (2000). Acute appendicitis in children: emergency department diagnosis and management. Annals of emergency medicine, 36(1), 39-51.

11. Sivit, C. J., Applegate, K. E., Stallion, A., Dudgeon, D. L., Salvator, A., Schluchter, M., ... \& Grisoni, E. R. (2000). Imaging evaluation of suspected appendicitis in a pediatric population: effectiveness of sonography versus CT. American Journal of Roentgenology, 175(4), 977-980.

12. Fahimi, J., Herring, A., Harries, A., Gonzales, R., \& Alter, H. (2012). Computed tomography use among children presenting to emergency departments with abdominal pain. Pediatrics, 130(5), e1069-e1075 